\title{
Back to the future: new techniques show that forgotten phosphorylation sites are present in contractile proteins of the heart whilst intensively studied sites appear to be absent
}

\author{
Steven B. Marston · Jeffrey W. Walker
}

Received: 26 June 2009/Accepted: 7 July 2009/Published online: 25 July 2009

(c) The Author(s) 2009. This article is published with open access at Springerlink.com

It is well established that several key proteins of the contractile apparatus are phosphoproteins. This includes myosin binding protein $\mathrm{C}$ (MyBP-C), troponin $\mathrm{T}$ and troponin I. It is also generally accepted that phosphorylation of these proteins alters their functional properties and that this modulation of function through the action of kinases and phosphatases plays a role in tuning the contractile apparatus to physiological demands. The prime example of this is phosphorylation of troponin I and MyBP-C by PKA as part of inotropic and lusitropic responses to $\beta$-adrenergic stimulation.

What is considerably less certain is the 'where' and 'when' of these phosphorylations. The sites of contractile protein phosphorylation by kinases such as PKA and PKC have been determined in vitro and the consequences of changes in phosphorylation at these sites have been investigated by site directed mutagenesis and transgenic mouse models. What has not been determined is whether these predominantly in vitro measurements in purified proteins and in rodent models have any relevance to the human heart in vivo.

Recently several papers have addressed this question with new techniques and the results have been surprising and somewhat disconcerting. Phosphorylation in human and rat troponin I and troponin $\mathrm{T}$ has recently been studied using electrospray ionisation and Fourier transform mass spectrometry (Zabrouskov et al. 2008; Sancho Solis et al.

\footnotetext{
S. B. Marston ( $\square)$

Imperial College London, London SW3 6LY, UK

e-mail: s.marston@imperial.ac.uk

J. W. Walker

University of Arizona College of Medicine, Tucson, AR 85724,

USA

e-mail: jwwalker@arizona.edu
}

2008). The great advantage of this method is that the mass of the intact protein can be determined with an accuracy high enough to unambiguously assign the isoform and the nature and number of post-translational modifications, whilst subsequent splitting of the molecular ion can define where in the sequence the modifications are located. When applied to troponin this method reveals the precise patterns of troponin I and troponin $\mathrm{T}$ phosphorylation isolated from heart muscle.

According to current literature troponin I is phosphorylated in vitro by PKA at Ser22 and 23, by PKC at Ser41, Ser43 and Thr142 and by PAK1 at Ser149 (Noland et al. 1989; Buscemi et al. 2002; Layland et al. 2005) [for consistency we use the numbering of the native human cardiac troponin I sequence in which the $\mathrm{N}$-terminal methionine is removed during post-translational processing (Zabrouskov et al. 2008)]. Measurements of total phosphorylation indicate that 1.6-2.3 mol of $\mathrm{Pi}$ are incorporated per mole of troponin I but where is the troponin I phosphorylated (Messer et al. 2009)? One might naturally assume that the troponin I sites phosphorylated in vitro are those phosphorylated in vivo, but under what conditions? Thus far the new mass spectrometry techniques have been applied to whole troponin, purified from healthy unstimulated hearts by antibody affinity methods, to reveal basal phosphorylation patterns (Messer et al. 2007; Sancho Solis et al. 2008). Analysis of human troponin I confirms that Ser22 and 23 are phosphorylated and constitute approximately half of the phosphorylation sites whilst a complementary technique of phosphate affinity SDS-PAGE shows that Ser22 + Ser23 constitute closer to 2/3 of phosphorylation sites (Messer et al. 2009). Mass spectrometry further reveals the location of these and other prominent sites and somewhat surprisingly shows no sign of phosphorylation at Ser41, Ser43, Thr142 or Ser149. Instead, the remaining $1 / 2$ to $1 / 3$ of 
phosphorylation is at Ser76 or Thr77 (the mass spectrometry techniques do not yet distinguish between the two). This may not be a truly new site: curiously enough, Ser76 was listed as one of the sites phosphorylated by PKC in the earliest publications but it has been forgotten over the years (Noland et al. 1989). This site in human troponin I may have been a 'casualty' of the recent emphasis on rodent models in which Ser76 is conserved but Thr77 is not. Meanwhile, in vitro research and increasing use of transgenic mouse models has concentrated on elucidating the function of the other sites phosphorylated by PKC (Layland et al. 2005), which are now found to be largely absent in the heart at least under basal conditions. These new studies are reminiscent of studies of cardiac troponin by ${ }^{31} \mathrm{P}-\mathrm{NMR}$ in which only Ser22 and Ser23 were found to be phosphorylated, either individually or together (Beier et al. 1988; Jaquet et al. 1993). Among the strengths of ${ }^{31} \mathrm{P}-\mathrm{NMR}$ is its ability to probe conformation and environment of phosphate moieties, however, its inability to detect low levels of phosphorylation left open the possibility that key basal phosphorylation sites were missed in this analysis. The new mass spectrometry and phosphate affinity SDS-PAGE techniques are much more sensitive and still do not reveal basal phosphorylation of widely studied PKC sites in human, rat or mouse heart.

A similar re-emergence of a forgotten site occurs with troponin $\mathrm{T}$. The earliest reports, based on the now-obsolete Edman degradation approach to sequencing, identified Ser1 of cardiac troponin $\mathrm{T}$ as a phosphorylation site (and Thr1 in skeletal muscle; Gusev et al. 1980, 1983). This phosphorylation was apparently constitutive and therefore not thought to be of importance in beat-to-beat physiological regulation of the heart. Early ${ }^{31} \mathrm{P}-\mathrm{NMR}$ measurements described two phosphorylation signals for bovine troponin $\mathrm{T}$, one at Ser1 and another in the C-terminal $\mathrm{CNBr}$ fragment (residues 181-285, bovine sequence) (Swiderek et al. 1990). Recent investigations of human heart samples have confirmed that troponin $\mathrm{T}$ phosphorylation is virtually constant in normal and pathological muscle where troponin I phosphorylation levels vary enormously (van der Velden et al. 2003; Messer et al. 2007). A number of other sites in troponin $\mathrm{T}$, phosphorylated by PKC and CaMKII in vitro have been identified and their regulatory significance was studied by site-directed mutagenesis. The phosphorylation of Thr203 by PKC has been particularly thoroughly investigated since phosphorylation at this site produced measurable functional effects (Sumandea et al. 2003).

The mass spectrometry measurements on heart troponin bring us right back to the initial studies. Troponin $\mathrm{T}$ in rat heart muscle is $100 \%$ monophosphorylated, the site of phosphorylation is almost certainly Ser1 and all possible sites beyond amino acid 30 have been essentially excluded, at least under basal conditions (Sancho Solis et al. 2008). In a previous report, using Pro-Q Diamond phosphoprotein stain, troponin $\mathrm{T}$ phosphorylation in human heart was calculated to be up to $3 \mathrm{~mol} \mathrm{Pi}$ per mole. This is probably in error due to the anomalous behaviour of the phosphatechelating Pro-Q Diamond probe when phosphorylation is on the N-terminal amino acid (Messer et al. 2007, 2009). On the other hand, there may be significant species differences that need to be evaluated systematically.

The final case is MyBP-C. Initially 3 PKA sites were defined in the cardiac-specific linker between domains $\mathrm{C} 1$ and $\mathrm{C} 2$ based on sequence analysis and site-directed mutagenesis (Gautel et al. 1995). MyBP-C is too big to be analysed fully intact by current ESI methods but it has been studied by $2 \mathrm{D}$ electrophoresis of heart muscle myofibrils, followed by mass spectrometry analysis of proteolytic fragments (Yuan et al. 2006; Duncker et al. 2009). The presence of 11 spots on 2D electrophoresis, many of which stain positive for Pro-Q Diamond, established that there are multiple phosphorylated species and a quantitative measurement indicated 4-5 mol Pi per mol MyBP-C in human heart (Jacques et al. 2008). In fact, mass spectrometry identified five different sites phosphorylated and only two of these corresponded to the proposed PKA sites.

This new data should initiate a radical rethink of how contractile proteins are modulated by phosphorylation in the heart. The in vitro approach of identifying sites phosphorylated in pure contractile proteins and their analysis by site-directed mutagenesis is useful but should not stand alone. It is also essential to know the sites phosphorylated in the intact heart and how they change with physiological and pathological changes to their environment (Marston and deTombe 2008). Perhaps the PKC and PAK1 sites found to be unphosphorylated in the basal 'healthy' state are quite dynamic and this characteristic needs to be carefully considered in efforts to 'trap' phosphorylation on these sites in vivo. The recent publications show we now have powerful tools to unambiguously determine the phosphorylation status of these proteins isolated from single rodent hearts and from human heart biopsies.

Whilst the PKA sites at serines 22 and 23 of troponin I and their modulation of $\mathrm{Ca}^{2+}$-sensitivity and $\mathrm{Ca}^{2+}$-dissociation kinetics seems to be a sound hypothesis, the role of PKC, PAK1, AMP kinase and CaMKII phosphorylation in vivo is now most uncertain. Direct measurements do not support the hypothesis that increased PKC activity in failing heart (Bowling et al. 1999) leads to increased phosphorylation of troponin I or troponin $\mathrm{T}$ : in fact total phosphorylation levels in troponin I and MyBP-C are reduced in heart failure suggesting that PKC may contribute to down-regulation of the beta-adrenergic system and may activate phosphatases instead (Neumann et al. 1997; Messer et al. 2007).

It seems we now know less than we thought we did about the role of post-translational modifications in 
modulating cardiac contractile function. This may be partly due to the oversimplified view of phosphorylation as a regulatory signal that switches between low, intermediate and high levels of occupancy on a few special sites as is now widely accepted for the Ser22/23 sites in cardiac troponin I. We should be heartened by the fact that we now have the tools that will ensure future research can be directed towards elucidating the physiological functions of those phosphorylation sites that are dynamically phosphorylated by specific and interesting environmental circumstances. The new work focuses attention on sites that show substantial basal phosphorylation, but continued interest in other phosphorylation sites will depend upon being able to demonstrate their occupancy in vivo under physiologically or pathologically relevant conditions.

Open Access This article is distributed under the terms of the Creative Commons Attribution Noncommercial License which permits any noncommercial use, distribution, and reproduction in any medium, provided the original author(s) and source are credited.

\section{References}

Beier N, Jaquet K, Schnackerz K, Heilmeyer LM (1988) Isolation and characterization of a highly phosphorylated troponin from bovine heart. Eur J Biochem 176:327-334

Bowling N, Walsh RA, Song G, Estridge T, Sandusky GE, Fouts RL, Mintze K, Pickard T, Roden R, Bristow MR, Sabbah HN, Mizrahi JL, Gromo G, King GL, Vlahos CJ (1999) Increased protein kinase $\mathrm{C}$ activity and expression of $\mathrm{Ca} 2+$-sensitive isoforms in the failing human heart. Circulation 99:384-391

Buscemi N, Foster DB, Neverova I, Van Eyk JE (2002) p21-activated kinase increases the calcium sensitivity of rat triton-skinned cardiac muscle fiber bundles via a mechanism potentially involving novel phosphorylation of troponin I. Circ Res 91:509-516

Duncker D, Boontje NM, Merkus D, Versteilen A, Krysiak J, Mearini G, El-Armouche A, de Beer V, Lamers J, Carrier L, Walker L, Linke W, Stienen G, van der Velden J (2009) Prevention of myofilament dysfunction by $\beta$-blocker therapy in postinfarct remodeling. Circ Heart Fail 2:233-242

Gautel M, Zuffardi O, Freiburg A, Labeit S (1995) Phosphorylation switches specific for the cardiac isoform of myosin binding protein-C: a modulator of cardiac contraction? EMBO J 14:1952-1960

Gusev NB, Dobrovolskii AB, Severin SE (1980) Isolation and some properties of troponinT kinase from rabbit skeletal muscle. Biochem J 189:219-226

Gusev NB, Barskaya NV, Verin AD, Duzhenkova IV, Khuchua ZA, Zheltova AO (1983) Some properties of cardiac troponin T structure. Biochem J 213:123-129

Jacques A, Copeland O, Messer A, Gallon C, King C, McKenna W, Tsang V, Marston S (2008) Myosin binding protein C phosphorylation in normal, hypertrophic and failing human heart muscle. J Mol Cell Cardiol 45:209-216

Jaquet K, Korte K, Schnackerz K, Vyska K, Heilmeyer LM Jr (1993) Characterization of the cardiac troponin I phosphorylation domain by $31 \mathrm{P}$ nuclear magnetic resonance spectroscopy. Biochemistry 32:13873-13878

Layland J, Solaro RJ, Shah AM (2005) Regulation of cardiac contractile function by troponin I phosphorylation. Cardiovasc Res 66:12-21

Marston S, deTombe P (2008) Point/counterpoint. Troponin phosphorylation and myofilament $\mathrm{Ca} 2+$-sensitivity in heart failure: increased or decreased? J Mol Cell Cardiol 45:603-607

Messer AE, Jacques AM, Marston SB (2007) Troponin phosphorylation and regulatory function in human heart muscle: Dephosphorylation of Ser23/24 on troponin I could account for the contractile defect in end-stage heart failure. J Mol Cell Cardiol 42:247-259

Messer A, Gallon C, Marston S (2009) Analysis of cardiac myofibrillar troponin I phosphorylation in normal and failing human hearts using phos-tags. Biophys J 96:501a

Neumann J, Eschenhagen T, Jones LR, Linck B, Schmitz W, Scholz H, Zimmermann N (1997) Increased expression of cardiac phosphatases in patients with end-stage heart failure. J Mol Cell Cardiol 29:265-272

Noland T, Raynor R, Kuo J (1989) Identification of sites phosphorylated in bovine cardiac troponin $\mathrm{I}$ and troponin $\mathrm{T}$ by protein kinase $\mathrm{C}$ and comparative substrate activity of synthetic peptides containing the phosphorylation sites. J Biol Chem 264:2077820785

Sancho Solis R, Ge Y, Walker JW (2008) Single amino acid sequence polymorphisms in rat cardiac troponin revealed by top-down tandem mass spectrometry. J Muscle Res Cell Motil 29:203-212

Sumandea MP, Pyle WG, Kobayashi T, de Tombe PP, Solaro RJ (2003) Identification of a functionally critical protein kinase C phosphorylation residue of cardiac troponin T. J Biol Chem 278:35135-35144

Swiderek K, Jaquet K, Meyer HE, Schachtele C, Hofmann F, Heilmeyer LM Jr (1990) Sites phosphorylated in bovine cardiac troponin $\mathrm{T}$ and I. Characterization by 31P-NMR spectroscopy and phosphorylation by protein kinases. Eur $\mathrm{J}$ Biochem 190:575-582

van der Velden J, Papp Z, Zaremba R, Boontje NM, de Jong JW, Owen VJ, Burton PB, Goldmann P, Jaquet K, Stienen GJ (2003) Increased $\mathrm{Ca} 2+$-sensitivity of the contractile apparatus in endstage human heart failure results from altered phosphorylation of contractile proteins. Cardiovasc Res 57:37-47

Yuan C, Guo Y, Ravi R, Przyklenk K, Shilkofski N, Diez R, Cole RN, Murphy AM (2006) Myosin binding protein C is differentially phosphorylated upon myocardial stunning in canine and rat hearts-evidence for novel phosphorylation sites. Proteomics 6:4176-4186

Zabrouskov V, Ge Y, Schwartz J, Walker JW (2008) Unraveling molecular complexity of phosphorylated human cardiac troponin I by top down electron capture dissociation/electron transfer dissociation mass spectrometry. Mol Cell Proteomics 7:18381849 